\title{
Malformación arteriovenosa pulmonar: Características clínicas, diagnóstico y rol del tratamiento quirúrgico en pacientes tratados con cirugía resectiva pulmonar
}

\begin{abstract}
ROBERTO GONZÁLEZ L.***, CLAUDIO CIFUENTES V.*, GERARDO MORDOJOVICH R.*,**, RAFAEL PRATS M.*,**, RAIMUNDO SANTOLAYA C.*,** y PATRICIO RODRÍGUEZ D.***
\end{abstract}

Pulmonary arteriovenous malformation: Clinical features, diagnosis and role of surgical management in patients with lung resection surgery

Background: Pulmonary arteriovenous malformations (PAVM) are rare and surgery has a role only in selected cases. Our objectives are to describe clinical features, diagnostic methods and role of surgical treatment in patients with PAVM. Methods: Retrospective review of all patients with PAVM, in whom surgery was performed in our institution, from February 2005 to February 2010. The follow up controls were done through physician or telephone contact. Results: 8 patients, six females (3:1), aged between 16-68 years were analyzed. Most common signs and symptoms were dyspnea, cyanosis and clubbing. Right lower lobe was the most frequent location. Four had multiple PAVM and four met criteria for Rendu-Osler-Weber disease. Six patients had polycythemia and two anemia. Radiography was abnormal in all and computed tomography defined anatomy in seven. Angiography was performed in three, two had contrasted echocardiography and four had scintigraphy. Most common surgical treatment was lobectomy. Indications for surgery were the size of PAVM in five cases. failure of embolization in two and one because of intra-operative findings, without a previous diagnosis. One had postoperative bleeding. Discharge was between day 2 and 10 days after surgery. There was no mortality. At their last control all patients were asymptomatic. Conclusion: PAVM presents a wide and varied range of clinical and anatomical findings. They can cause major symptoms and serious complications, which justify their treatment. The preoperative study is based primarily on demonstrating the shunt and determining the anatomical characteristics of the lesion. In selected cases lung resection surgery is indicated.

Key words: Arteriovenous malformations; arteriovenous fistula; lung; thoracic surgery; telangiectasia, hereditary hemorrhagic.

\section{Resumen}

Introducción: Las malformaciones arteriovenosas pulmonares (MAVP) son infrecuentes y la cirugía tiene un rol en casos seleccionados. Objetivos: Describir las características clínicas, métodos diagnósticos y rol del tratamiento quirúrgico en pacientes tratados con cirugía por MAVP. Material y Método: Revisión retrospectiva, incluyendo todos los pacientes con diagnóstico de MAVP en quienes se realizó cirugía en el Instituto Nacional del Tórax, desde febrero de 2005 a febrero de 2010. El seguimiento fue por control médico o contacto telefónico. Resultados: Se analizó 8 pacientes, seis mujeres (relación 3:1), edad entre 16 y 68 años. Los sintomas y signos más frecuentes fueron: disnea, cianosis y acropaquia. La localización más frecuente fue el lóbulo inferior derecho. Cuatro pacientes tenían MAVP múltiples y cuatro cumplian con criterios de Enfermedad de Rendu-Osler-Weber. Seis tenian policitemia y dos anemia. La radiografía fue anormal en todos los casos y la tomografía computada

* Sección de Cirugía de Tórax, Servicio Médico Quirúrgico Respiratorio, Instituto Nacional del Tórax.

** Departamento de Cirugía, Campus Oriente, Facultad de Medicina, Universidad de Chile.

No existen conflictos de interés ni apoyo financiero. 
definió la anatomía en siete. Angiografía se realizó en tres casos. Dos tenían ecocardiograma con contraste y cuatro cintigrama. La cirugía más frecuente fue la lobectomía. La indicación de cirugía fue: tamaño de la MAVP en cinco, falla de embolización en dos y hallazgo intra operatorio en uno, intervenido por otra razón. Uno presentó hemorragia post-operatoria. La estadía post-operatoria fue entre 2 y 10 días. No hubo mortalidad. En el último control todos los pacientes estaban asintomáticos. Conclusión: Las MAVP pueden presentarse en un amplio espectro clínico y anatómico. Pueden generar sintomas y complicaciones graves, por esto se recomienda tratarlas. El estudio pre-operatorio se basa en demostrar el shunt y en determinar las características anatómicas de la lesión. En algunos casos seleccionados está indicada la cirugía resectiva pulmonar.

Palabras clave: Malformación arteriovenosa; fistula arteriovenosa; pulmón; cirugía torácica; telangectasia hereditaria hemorrágica.

\section{Introducción}

La comunicación anómala entre ramas de la arteria y vena pulmonar, sin paso de sangre por el lecho capilar pulmonar y con el consiguiente cortocircuito, es lo que conocemos como fístula arteriovenosa pulmonar o mejor llamada malformación arteriovenosa pulmonar (MAVP).

La primera publicación sobre MAVP corresponde a Churton en 1897, en que describe los hallazgos post mortem de un niño de 12 años con MAVP bilateral ${ }^{1}$. Wilkens en 1917, en la necropsia de una mujer de 23 años, describe como causa de muerte un hemotórax secundario a la rotura de una MAVP (la mujer presentaba cianosis, acropaquia, telangectasias y soplo axilar bilateral $)^{2}$. Rodees comunica en 1938 la asociación entre telangectasias y MAVP'3 31939 Smith y col, publican el primer caso con diagnóstico clínico de MAVP en un hombre de 40 años, que presentaba cianosis, acropaquia, soplo y policitemia ${ }^{4}$.

Las primeras comunicaciones de tratamiento quirúrgico de MAVP datan de los años cuarenta. Shenstone en forma separada de Hepburn y col, comunican casos de MAVP tratados con neumonectomía ${ }^{5,6}$. Desde esa fecha la cirugía se mantuvo como único tratamiento, sin embargo, a fines de los setenta, se conocen los primeros casos de tratamiento por embolización percutánea.

Las MAVP son infrecuentes, la incidencia es 2-3 casos por cada 100.000 habitantes y son más frecuentes en el género femenino (relación 2:1). Entre el $65 \%$ a $70 \%$ se presentan en pacientes con telangectasia hemorrágica hereditaria o Enfermedad de Rendu-Osler-Weber (EROW) ${ }^{7-12}$.

Las MAVP son de origen congénito en la inmensa mayoría de los casos. Entre las formas secundarias descritas se encuentran: post traumática, infecciosa, estados avanzados de cirrosis hepática, post operatoria en cirugía torácica y post operatoria en cardiopatías congénitas complejas $^{7,8,13}$.

El tratamiento de las MAVP se recomienda en casos sintomáticos o cuando son mayores de 3 milímetros ${ }^{7-12}$. La embolización ha demostrado ser segura y obtener buenos resultados con las ventajas propias de los tratamientos percutáneos, sin embargo, la cirugía continúa teniendo un rol en casos seleccionados ${ }^{10-12,14,15}$.

Los objetivos de nuestra comunicación son describir las características clínicas, métodos diagnósticos y rol del tratamiento quirúrgico, en pacientes con MAVP y tratados con cirugía resectiva.

\section{Pacientes y Métodos}

Se realizó una revisión retrospectiva de bases de datos, de protocolos operatorios, registro de pabellón y de anatomía patológica del Instituto Nacional del Tórax.

Se incluyeron todos los pacientes con diagnóstico de MAVP, en quienes se realizó tratamiento quirúrgico y que tenían confirmación diagnóstica con anatomía patológica.

El período comprendió desde febrero de 2005 a febrero de 2010.

Se describen características clínicas, métodos diagnósticos, características peri operatorias, variables quirúrgicas, cirugía, morbilidad, mortalidad y seguimiento post operatorio. Para determinar asociación con EROW, el diagnóstico de esta última se basó en los criterios de Curaçao ${ }^{16}$ (Tabla 1). Para el seguimiento se citó a pacientes a control o se contactaron telefónicamente.

\section{Resultados}

Durante el período estudiado, en el Instituto Nacional del Tórax se realizó un total de 4.969 cirugías torácicas (excluidas las cardiovasculares). De éstas, 8 fueron cirugías resectivas pulmonares en pacientes con MAVP, lo que equivale al $0,16 \%$ del quehacer quirúrgico. 
Tabla 1. Criterios diagnósticos de telangiectasia hemorrágica hereditaria

(Síndrome de Rendu-Osler-Weber)

\section{Criterios de Curaçao ${ }^{16}$}

1. Epistaxis recurrente.

2. Telangectasias múltiples en localizaciones típicas (labios, cavidad oral, nariz, lechos subungeales, dedos).

3. Lesiones viscerales (pulmonares, hepáticas, cerebrales, gastrointestinales, medulares).

4. Historia familiar, un pariente de primer grado con Enfermedad de Rendu-Osler-Weber.

Tres criterios establecen diagnóstico, Dos criterios: diagnóstico probable.

En la Tabla 2 se muestran las principales características clínicas de los pacientes. La mayoría corresponden al género femenino (relación de $3: 1)$ y la edad presentó un rango entre los $16 \mathrm{y}$ 68 años.

Los síntomas y signos más frecuentes atribuibles a la MAVP fueron disnea, cianosis y acropaquia. En cuanto a la localización, el lugar más frecuente fue el lóbulo inferior derecho, no existiendo claro predominio de esta sobre otras ubicaciones.

La mitad tenían MAVP múltiples, de ellas, la mayoría ubicadas en un mismo lóbulo. Un paciente presentó MAVP bilateral, en el lado derecho la MAVP era milimétrica y se decidió seguimiento y en la del lado izquierdo que era de mayor tamaño, se realizó cirugía resectiva.

Dos pacientes tenían el antecedente de tratamiento percutáneo con embolización, uno corresponde a un intento frustro y en el otro se realizaron 5 embolizaciones persistiendo el shunt.

La mitad de los pacientes cumplían con criterios diagnósticos de EROW (tres de cuatro criterios de $\operatorname{Curaçao}^{16}$ ) y en uno que presentaba dos criterios, se consideró diagnóstico probable.

De estos pacientes, dos presentaban malformaciones arteriovenosas en otros territorios, una paciente tenía una cerebral que fue tratada con embolización después de un evento neurológico y otra paciente tenía una hepática que luego de evaluación por cirujanos digestivos y gastroenterólogos se decidió seguimiento.

En la Tabla 3 se muestran otros síntomas, signos y hallazgos de los pacientes (síntomas y signos que pueden ser atribuibles a otras patologías

Tabla 2. Características clínicas de 8 pacientes tratados quirúrgicamente por malformación arteriovenosa pulmonar

\begin{tabular}{|c|c|c|c|c|c|c|c|c|}
\hline Caso & Género & $\begin{array}{l}\text { Edad } \\
\text { (años) }\end{array}$ & $\begin{array}{l}\text { Síntomas y signos } \\
\text { atribuibles a MAVP }\end{array}$ & $\begin{array}{l}\text { Localización } \\
\text { MAVP }\end{array}$ & $\begin{array}{l}\text { Única o } \\
\text { múltiple }\end{array}$ & $\begin{array}{c}\text { Embolización } \\
\text { previa }\end{array}$ & EROW & $\begin{array}{l}\text { Otras } \\
\text { MAV }\end{array}$ \\
\hline 1 & M & 57 & Disnea & LSD & $\mathrm{Mu}$ & No & No & No \\
\hline 2 & $\mathrm{~F}$ & 68 & $\begin{array}{l}\text { Tos } \\
\text { Hemoptisis }\end{array}$ & LID & Un & $\begin{array}{l}1 \text { intento frus- } \\
\text { tro }\end{array}$ & No & No \\
\hline 3 & $\mathrm{~F}$ & 56 & $\begin{array}{l}\text { Disnea } \\
\text { Cianosis } \\
\text { Hemoptisis }\end{array}$ & LSD & Un & No & No & No \\
\hline 4 & $\mathrm{~F}$ & 16 & $\begin{array}{l}\text { Disnea } \\
\text { Cianosis } \\
\text { Hemoptisis } \\
\text { Acropaquia }\end{array}$ & LID & $\mathrm{Mu}$ & 5 veces & Sí & No \\
\hline 5 & $\mathrm{~F}$ & 16 & $\begin{array}{l}\text { Disnea } \\
\text { Cianosis } \\
\text { Acropaquia }\end{array}$ & LM & Un & No & Sí & Cerebro \\
\hline 6 & $\mathrm{~F}$ & 51 & $\begin{array}{l}\text { Disnea } \\
\text { Acropaquia }\end{array}$ & LII & $\mathrm{Mu}$ & No & Prob & No \\
\hline 7 & M & 19 & Disnea & LII, LID & $\mathrm{Mu}$ & No & Sí & No \\
\hline 8 & $\mathrm{~F}$ & 41 & $\begin{array}{l}\text { Disnea } \\
\text { Cianosis } \\
\text { Acropaquia }\end{array}$ & $\mathrm{LM}$ & Un & No & Sí & Hígado \\
\hline
\end{tabular}

$(\mathrm{M}=$ masculino; $\mathrm{F}$ = femenino; MAVP = malformación arteriovenosa pulmonar; LSD = lóbulo superior derecho; LID = lóbulo inferior derecho; LM = lóbulo medio; LII = lóbulo inferior izquierdo; $\mathrm{Mu}=$ múltiple; Un = única; EROW = enfermedad de Rendu Osler Weber; Prob = probable; MAV = malformación arteriovenosa). 
Tabla 3. Otros síntomas, signos y hallazgos en 8 pacientes tratados quirúrgicamente por malformación arteriovenosa pulmonar

\begin{tabular}{|lc|}
\hline Otros síntomas, signos y hallazgos & n de casos \\
Policitemia & 6 \\
Epistaxis & 5 \\
Rubor facial & 4 \\
Dolor torácico & 2 \\
Telangectasias & 3 \\
Anemia & 2 \\
Soplo cardíaco & 1 \\
\hline
\end{tabular}

como a la EROW). Dentro de los hallazgos destaca que seis pacientes tenían policitemia y que dos que tenían anemia presentaban hemoptisis.

En la Tabla 4 se resumen los métodos diagnósticos. La radiografía de tórax fue anormal en todos los casos (Figura 1) y la tomografía computada de tórax se consideró sugerente de MAVP en siete (Figura 2). La angiografía convencional se realizó en tres (Figura 3), dos de los cuales corresponden a los que se intentó embolización. La angio-tomografia computada permitió plantear el diagnóstico en siete y en la mayoría de ellos se pudo evaluar anatómicamente vasos aferente y eferente (Figura 4). Sólo dos pacientes tenían

Tabla 4. Métodos diagnósticos, sospecha clínica y diagnóstico pre operatorio en 8 pacientes tratados quirúrgicamente por malformación arteriovenosa pulmonar

\begin{tabular}{|c|c|c|c|c|c|c|c|c|}
\hline Caso & Rx tórax & TAC & $\begin{array}{c}\text { Angio } \\
\text { TAC }\end{array}$ & $\begin{array}{l}\text { Angio- } \\
\text { grafía }\end{array}$ & Ecocardiografía & $\begin{array}{c}\text { Cintigrama } \\
\text { perfusión }\end{array}$ & $\begin{array}{c}\text { Sospecha } \\
\text { clínica }\end{array}$ & $\begin{array}{c}\text { Diagnóstico } \\
\text { Pre op }\end{array}$ \\
\hline 1 & Anormal & No Sug & $\mathrm{N} / \mathrm{R}$ & $\mathrm{N} / \mathrm{R}$ & $\mathrm{N} / \mathrm{R}$ & $\mathrm{N} / \mathrm{R}$ & No & No \\
\hline 2 & Anormal & Sug & Sug & Sí & * Si, Sug shunt & $\mathrm{N} / \mathrm{R}$ & Sí & Sí \\
\hline 3 & Anormal & Sug & Sug & $\mathrm{N} / \mathrm{R}$ & Sí & Sí & Sí & Sí \\
\hline 4 & Anormal & Sug & Sug & Sí & Sí & Sí & Sí & Sí \\
\hline 5 & Anormal & Sug & Sug & $\mathrm{N} / \mathrm{R}$ & Sí & Sí & Sí & Sí \\
\hline 6 & Anormal & Sug & Sug & $\mathrm{N} / \mathrm{R}$ & Sí & Sí & Sí & Sí \\
\hline 7 & Anormal & Sug & Sug & $\mathrm{N} / \mathrm{R}$ & * Si, Sug shunt & $\mathrm{N} / \mathrm{R}$ & Sí & Sí \\
\hline 8 & Anormal & Sug & Sug & Sí & Sí & $\mathrm{N} / \mathrm{R}$ & Sí & Sí \\
\hline
\end{tabular}

$(\mathrm{Rx}=$ radiografia; $\mathrm{TAC}=$ tomografía computada; Preo op $=$ pre operatorio; $\mathrm{Sug}=$ sugerente; $\mathrm{N} / \mathrm{R}=$ no realizado; * = ecocardiografía con contraste de burbujas).

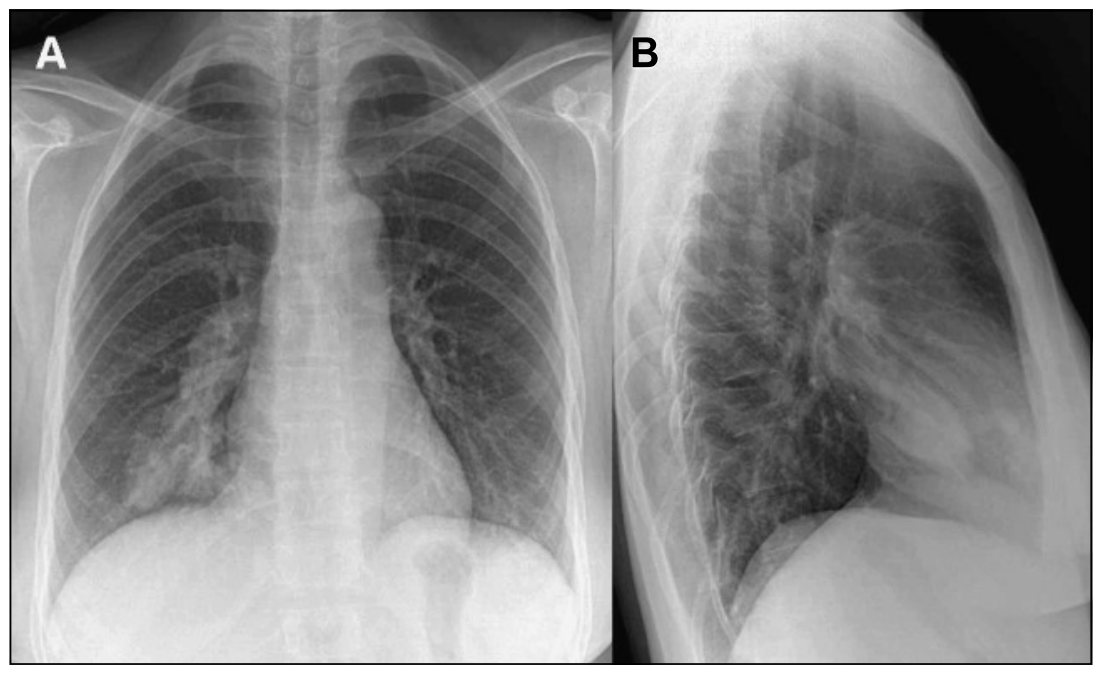

Figura 1. Radiografía de tórax postero-anterior y lateral (Caso 8): A y B. Se observa lesión en el lóbulo medio. 

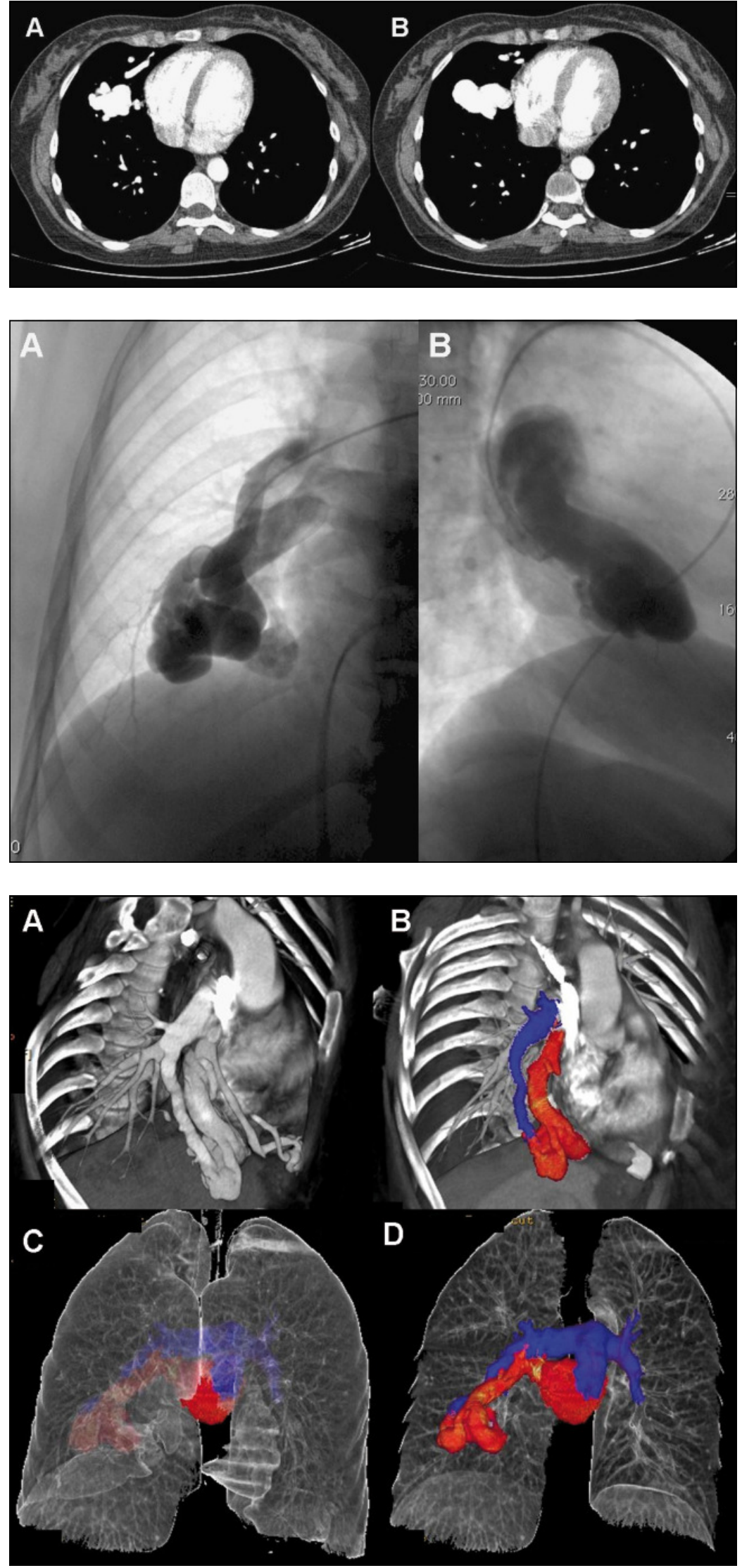

Figura 2. Tomografía computada de tórax (Caso 8): A y B. Se observa lesión compatible con malformación arteriovenosa en lóbulo medio.

Figura 3. Angiografía convencional, proyección posteroanterior y lateral (Caso 8): A y B. Se observa malformación arteriovenosa contrastada en lóbulo medio.

Figura 4. Angio-tomografía computada con reconstrucción tridimensional (Caso 8): A. Se observa malformación arteriovenosa en lóbulo medio, es posible identificar claramente vasos aferente y eferente. B. Malformación arteriovenosa contrastada con color para destacar vasos aferente y eferente

C y D. Malformación arteriovenosa y su relación con el parénquima pulmonar 
ecocardiograma con contraste de burbujas la que en ambos permitió identificar el shunt. El estudio de cuantificación de shunt se completó con cintigrama en la mitad de los pacientes.

La sospecha clínica en base a anamnesis, síntomas y signos se planteó en 7 casos. Una vez terminado el estudio pre operatorio se confirmó esta sospecha en todos. En el caso 1, nunca se planteó el diagnóstico pre operatorio de MAVP y correspondió a un hallazgo intra-operatorio que se confirmó con la anatomía patológica.
En la Tabla 5 se muestran características y variables peri operatorias. Destaca que en todos se constató mejoría inmediata en la saturación de $\mathrm{O}_{2}$ en el intra-operatorio, la que se mantuvo en el post-operatorio. La mayoría se abordó por toracotomía y la cirugía más frecuente fue la lobectomía.

En relación con el tamaño de las MAVP destaca que en general corresponden a lesiones grandes (Figura 5). Las mediciones corresponden al estudio anatomopatológico (Figura 6).

Tabla 5. Características y variables peri operatorias de 8 pacientes tratados quirúrgicamente por malformación arteriovenosa pulmonar

\begin{tabular}{|cccccccccc|}
\hline Caso & $\begin{array}{c}\text { Sat } \mathbf{O}_{2} \\
\text { \% } \\
\text { Pre op }\end{array}$ & Cirugía & $\begin{array}{c}\text { Abor- } \\
\text { daje }\end{array}$ & $\begin{array}{c}\text { Sat O } \\
\text { \% } \\
\text { Post op }\end{array}$ & Morbilidad & $\begin{array}{c}\text { Tamaño } \\
\text { MAVP } \\
\text { (mm) }\end{array}$ & $\begin{array}{c}\text { Estadía } \\
\text { Post op } \\
\text { (días) }\end{array}$ & $\begin{array}{c}\text { Segui- } \\
\text { miento } \\
\text { (meses) }\end{array}$ & $\begin{array}{c}\text { Estado } \\
\text { actual }\end{array}$ \\
\hline 1 & 92 & Segmentec & VATS & 99 & No & $25 \times 10 \times 10$ & 2 & $6 *$ & Desconocido \\
\hline 2 & 92 & Lobec & Toraco & 98 & No & $50 \times 50 \times 50$ & 8 & 50 & Asintomático \\
\hline 3 & 77 & Lobec & Toraco & 97 & No & $60 \times 40 \times 40$ & 10 & $6 *$ & Desconocido \\
4 & 69 & Lobec & Toraco & 98 & $\begin{array}{c}\text { Reop sang } \\
\text { Dehis hda op }\end{array}$ & $35 \times 20 \times 10$ & 10 & 38 & Asintomático \\
\hline 5 & 76 & Lobec & Toraco & 99 & No & $90 \times 40 \times 30$ & 5 & 20 & Asintomático \\
\hline 6 & 85 & Lobec & Toraco & 99 & No & $60 \times 60 \times 60$ & 5 & 20 & Asintomático \\
7 & 88 & Segmentec & Toraco & 95 & No & $50 \times 20 \times 15$ & 9 & 14 & Asintomático \\
8 & 82 & Lobec & Toraco & 99 & No & $120 \times 70 \times 30$ & 6 & 6 & Asintomático \\
\hline
\end{tabular}

(Sat $=$ saturación; Preo op $=$ pre operatorio; Post op $=$ post operatorio; MAVP $=$ malformación arteriovenosa pulmonar; $\mathrm{mm}=$ milímetros; Segmentec $=$ segmentectomía $;$ Lobec $=$ lobectomía; VATS $=$ cirugía torácica video asistida; Toraco = toracotomía; Reop sang = reoperación por sangrado; Dehis hda op $=$ dehiscencia de herida operatoria; $*=$ asintomático hasta el último control).

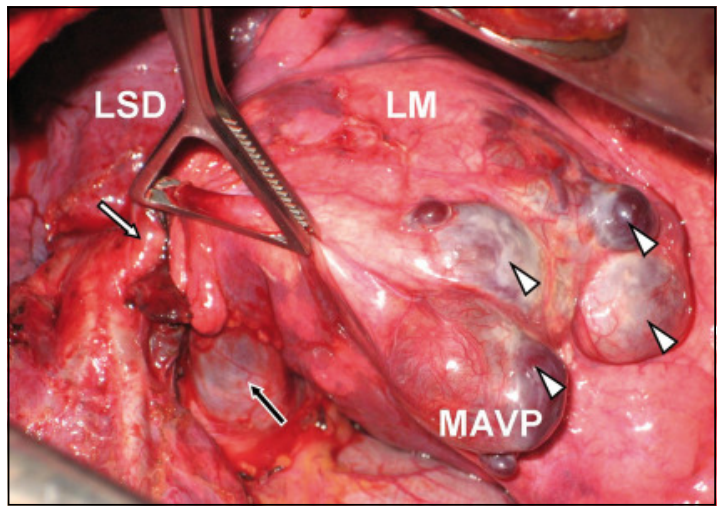

Figura 5. Fotografia intra-operatoria (Caso 8): Se observa aspecto de la malformación arteriovenosa en el lóbulo medio (Flecha blanca $=$ vaso aferente; flecha negra $=$ vaso eferente; cabezas de flecha $=$ malformación arteriovenosa en la superficie de lóbulo medio; LSD = lóbulo superior derecho; LM = lóbulo medio; MAVP = malformación arteriovenosa pulmonar).

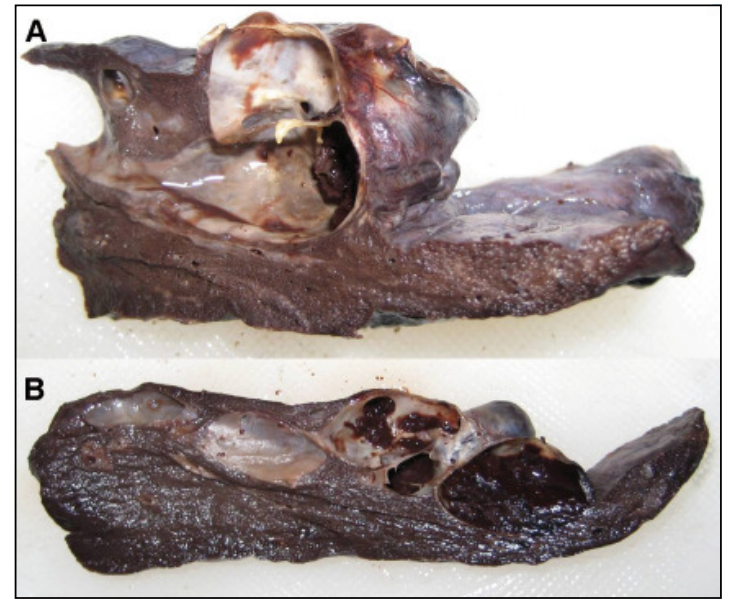

Figura 6. Macroscopía en el estudio anatomopatológico (Caso 8): A. Se observan amplios lechos vasculares de la malformación arteriovenosa. B. Algunos lechos vasculares de la malformación arteriovenosa con trombos. 
La indicación de cirugía fue por el tamaño de la MAVP en 5 pacientes (mayor o igual a 50 milímetros en uno de sus diámetros), por falla del tratamiento percutáneo en 2 (casos 2 y 4$)$ y hallazgo intra-operatorio en uno (caso 1).

Un paciente presentó hemorragia post-operatoria que necesitó reexploración y evolucionó con dehiscencia parcial de la herida operatoria. $\mathrm{La}$ estadía post-operatoria fue entre 2 y 10 días. No hubo mortalidad.

Todos los pacientes tienen un seguimiento de al menos 6 meses post- operatorio. Al momento del último control todos se encontraban asintomáticos de la cirugía de MAVP y en dos casos no fue posible el contacto para conocer estado actual.

\section{Comentario}

Nuestro hospital, por sus características de centro docente-asistencial y centro de referencia, corresponde sin duda al lugar donde más cirugías torácicas se realizan en nuestro país, lo que conlleva a que exista una importante experiencia en un amplio espectro de patologías de resolución quirúrgica. Pese a esto, la cirugía resectiva pulmonar como tratamiento de las MAVP es infrecuente y corresponde a un mínimo porcentaje de nuestro quehacer quirúrgico, por lo que la experiencia en este tipo de cirugía es limitada. Resulta entonces interesante comentar esta serie de pacientes, en quienes la cirugía jugó un papel como tratamiento.

Las manifestaciones clínicas de las MAVP son variadas y dependen principalmente del tamaño y de la cuantía del shunt. Se pueden presentar desde casos asintomáticos hasta pacientes con hipoxemia grave o complicaciones como accidentes neurológicos o hemorragias exanguinantes ${ }^{7,8,12}$. Todos los pacientes de nuestra serie presentaban síntomas atribuibles a la MAVP. En uno inició el estudio y se demostró la MAVP luego de un accidente neurológico en la infancia.

A nivel pulmonar las manifestaciones clínicas de las MAVP son: hipoxemia como consecuencia del shunt derecha-izquierda, lo que produce disnea, cianosis y policitemia; embolias paradójicas sistémicas principalmente a nivel cerebral (abscesos y accidentes isquémicos cerebrales) y sangrado (hemoptisis y hemotórax) por rotura ${ }^{8,12}$. La hemoptisis y el hemotórax espontáneo masivo son una presentación infrecuente, que ha sido descrita principalmente en mujeres embarazadas, en quienes el tamaño de las MAVP pueden experimentar un importante aumento ${ }^{10-12,17-20,21}$.

Clínicamente las MAVP se manifiestan más frecuentemente después de la tercera década de la vida y especialmente entre la quinta y sexta, ya mientras que las manifestaciones clínicas en las primeras décadas son relacionadas con los otros síntomas de la EROW como epistaxis y telangectasias mucocutáneas ${ }^{10,11}$.

Como entre el $65 \%$ a $70 \%$ estas manifestaciones se presentan en pacientes con EROW y en nuestra serie de ocho casos, cuatro tenían criterios diagnósticos y uno presentaba diagnóstico probable. Por ello es conveniente señalar algunas características de esta enfermedad.

La EROW es una enfermedad genética autosómica dominante, caracterizada por epistaxis recurrente, telangectasias mucocutáneas y malformaciones vasculares viscerales ${ }^{10,11,22}$. Su diagnóstico clínico se basa en los criterios de Curaçao $^{16,22}$. Deben cumplirse tres de cuatro para establecer diagnóstico y dos para considerarlo probable (Tabla 1).

Si bien es cierto la mayoría de los pacientes con MAVP presentan EROW, sólo entre el 15 al $35 \%$ de los pacientes con EROW presentan MAVP $^{7-12,22}$.

Las MAVP frecuentemente se encuentran en los lóbulos inferiores (60-95\%), pudiendo ser múltiples (35-65\%), uni o bilaterales y no existiendo predominio de $\mathrm{lado}^{7-12,22}$. En nuestra serie hubo más casos en el lóbulo inferior derecho pero no existió predominio claro respecto a la ubicación. Por otro lado, la mitad presentaban lesiones múltiples, que en la mayoría se ubicaban en un solo lóbulo.

Ante la sospecha clínica se debe objetivar el shunt y completar el estudio con imágenes que permitan una adecuada evaluación anatómica $8,22,23$.

La estimación del shunt mediante el método de $\mathrm{O}_{2}$ al $100 \%$ por 20 minutos permite una evaluación no invasiva de éste y si el shunt es mayor al $5 \%$ se debe complementar el estudio con otros métodos ${ }^{8}$.

El diagnóstico ecocardiográfico con contraste (burbujas) es útil especialmente en la pesquisa, permitiendo objetivar el shunt y evaluar la presencia y cuantía de hipertensión pulmonar. Se considera que es el método de elección en pesquisa (screening) de MAVP en pacientes sospechosos, como los portadores de $\mathrm{EROW}^{8,11,12,22-24}$.

El cintigrama de perfusión pulmonar es otro excelente método para cuantificar adecuadamente el shunt, sin embargo, tiene la desventaja que no aporta información anatómica relevante , $^{8,11,12,23}$.

La radiografía convencional es anormal hasta en el $98 \%$ de los casos, se observan masas ovaladas especialmente en lóbulos inferiores únicas, 
múltiples o bilaterales ${ }^{8-12,25}$. Todos los pacientes de esta serie presentaron alteraciones radiograficas.

La angiografía tradicional se consideró por mucho tiempo el gold standard indiscutido y un examen obligatorio en el diagnóstico de MAVP. Permite una adecuada visualización anatómica y de estructuras vasculares, además, juega un rol fundamental en la planificación y realización de la terapia endovascular. Como método diagnóstico en los últimos años se ha visto desplazado por la tomografía computada helicoidal $1^{7,12,22,25-27}$. En esta serie de pacientes quirúrgicos sólo se utilizó en tres casos y en dos de ellos en el contexto de tratamiento percutáneo frustro.

La tomografía computada helicoidal permite evaluar la ubicación, tamaño, vasos aferentes y eferentes. El uso de contraste y reconstrucciones tridimensionales permiten una excelente evaluación anatómica y la posibilidad de pesquisar otras posibles lesiones. Sin duda hoy constituye el examen de elección en el estudio anatómico de ellas $8,12,22,26,27$.

El uso de resonancia magnética es menor en comparación con la tomografía computada helicoidal, debido a que no existe mayor diferencia en cuanto a la capacidad de mostrar anatómicamente las lesiones, además de su menor disponibilidad y mayor costo ${ }^{8}$.

Se recomienda tratamiento en las MAVP sintomáticas o mayores de 3 milímetros ${ }^{7-12,22}$. Las razones se resumen en los siguientes puntos:

- Prevención de embolias paradójicas, especialmente que puedan dejar secuelas neurológicas. El riesgo estimado de sufrir un accidente neurológico encefálico en pacientes con MAVP igual o mayor de 3 milímetros es de 1,5\% anual.

- Prevención de hemoptisis y/o hemotórax masivo especialmente en mujeres con posibilidad de embarazo.

- Tratamiento de la hipoxemia y síntomas propios del shunt de derecha a izquierda como disnea y cianosis.

Desde fines de los '70 se han publicado múltiples series de pacientes tratados con embolización percutánea. Los avances tecnológicos en el desarrollo y diseño de nuevos materiales (coils, diversas partículas de embolización, balones, guías, etc), junto con el perfeccionamiento de los métodos de imagen han llevado a que este tipo de tratamiento obtenga buenos resultados. El éxito inmediato de estos procedimientos en grupos entrenados supera el 95\% $\%^{7,10-12,14,15}$.

El porcentaje de recanalización según los gru- pos de trabajo y el tiempo de seguimiento varía entre 5 y $57 \%$, las complicaciones de esta técnica si bien es cierto son infrecuentes, pueden llegar a ser graves como: embolia aérea, embolización paradójica de coils, sangrado (hemotórax) y rotura cardíaca. Entre otras complicaciones descritas están: migración de coils, infarto pulmonar, lesiones vasculares, trombosis, dolor pleurítico y derrame pleural ${ }^{14,15}$. En dos de nuestros pacientes se intentó tratamiento percutáneo sin éxito. Incluso uno de ellos tuvo 5 intentos.

Los buenos resultados, la baja morbimortalidad obtenida y las ventajas propias de los tratamientos percutáneos, han llevado a que este tratamiento sea el de elección en la inmensa mayoría de las MAVP ${ }^{8,10-12,22}$.

Antes del tratamiento percutáneo, la cirugía se consideró el único tratamiento de las MAVP. Diferentes técnicas quirúrgicas han sido empleadas, entre estas: ligadura de fístula, resecciones atípicas como cuñas y fistulectomía, resecciones anatómicas como segmentectomía, lobectomía y neumonectomía ${ }^{7,28-33}$. En pacientes con grandes malformaciones bilaterales se ha realizado incluso trasplante pulmonar ${ }^{34,35}$. En algunos casos (por ejemplo en lesiones periféricas), la cirugía torácica video asistida (VATS) puede ser una buena opción de abordaje ${ }^{28}$. Uno de nuestros pacientes fue operado por esta vía.

La mortalidad operatoria es cercana a cero y las complicaciones son infrecuentes, correspondiendo a las complicaciones propias de este tipo de cirugía (hemorragia, infección, fuga aérea, etc.) ${ }^{7}$. La recanalización de lesiones es casi nula y cuando se produce más bien corresponde a crecimiento y desarrollo de otras lesiones. El alivio sintomático post operatorio es muy evidente. Pick y colaboradores, en la serie de la Clínica Mayo en 20 años de experiencia comunican 30 casos operados y de estos más del $90 \%$ presentan desaparición de los síntomas ${ }^{7}$, en nuestra serie todos se encontraban asintomáticos en el último control del seguimiento.

Hoy en día la cirugía como tratamiento de las MAVP está indicada cuando falla la embolización, cuando ésta no es posible de realizar, en situaciones de urgencia (rotura, sangrado) o en MAVP de localización única y de gran tamaño $0^{7,28-33,36}$.

\section{Conclusiones}

Como conclusiones podemos decir que: las MAVP pueden presentarse en un amplio espectro clínico y anatómico; pueden generar importantes 
síntomas y complicaciones graves, por lo que en general se recomienda su tratamiento; el estudio pre-operatorio se basa principalmente en demostrar el shunt y en determinar adecuadamente las características anatómicas de la lesión; el tratamiento de elección es percutáneo (endovascular) y en algunos casos la cirugía resectiva pulmonar está indicada.

\section{Bibliografía}

1.- CHURTON T. Multiple aneurysms of pulmonary artery. BMJ 1897; 1: 1223.

2.- WILKINS GD. Ein Fall von multiplen pulmonalis aneurysmen. Beitr Klinis Tuberk 1917; 39: 1-10.

3.- RHODES C B. Cavernous hemangiomas of lung with secondary polycitemia. JAMA 1938; 110: 1914-5.

4.- SMITH H L, HORTON B T. Arteriovenous fistula of the lung associated with polycythemia vera; report of case in which the diagnosis was made clinically. Am Heart J 1939; 18: 589-92.

5.- SHENSTONE N S. Experiences with total pneumonectomy. J Thorac Surg 1942; 11: 405.

6.- HEPBURN J, DAUPHINEE J A. Successful removal of hemangioma of lung followed by disappearance of polycythemia. Am J Med Sci 1942; 204: 681-7.

7.- PICK A, DESCHAMPS C, STANSON A W. Pulmonary arteriovenous fistula: presentation, diagnosis, and treatment. World J Surg 1999; 23: 1118-22.

8.- KHURSHID I, DOWNIE G H. Pulmonary arteriovenous malformation. Postgrad Med J 2002; 78: 191-7.

9.- LÓPEZ VIME R, DE MIGUEL DÍEZ J, JARA CHINARRO B, SALGADO SALINAS R, GÓMEZ SANTOS D, SERRANO IGLESIAS J A. [Diagnosis and treatment of pulmonary arteriovenous fistulas]. Arch Bronconeumol 2002; 38: 288-90.

10.- SHARATHKUMAR A A, SHAPIRO A. Hereditary haemorrhagic telangiectasia. Haemophilia 2008; 14: 1269-80

11.- BEGBIE M E, WALLACE G M, SHOVLIN C L. Hereditary haemorrhagic telangiectasia (Osler-Weber-Rendu syndrome): a view from the 21 st century. Postgrad Med J 2003; 79: 18-24.

12.- COTTIN V, DUPUIS-GIROD S, LESCA G, CORDIER J F. Pulmonary vascular manifestations of hereditary hemorrhagic telangiectasia (Rendu-Osler Disease). Respiration 2007; 74: 361-78.

13.- PLOCH PJ, DATTA S, THOMPSON JH, RAGHAVENDRAN K. Posttraumatic pulmonary arteriovenous fistula: is resection the procedure of choice? A case report and review of literature. J Trauma 2009; 66: 554-7.

14.- GUPTA S, FAUGHNAN M E, BAYOUMI A M. Embolization for pulmonary arteriovenous malformation in hereditary hemorrhagic telangiectasia: a decision analysis. Chest 2009; 136: 849-58.
15.- LETOURNEAU-GUILLON L, FAUGHNAN ME, SOULEZ G, GIROUX MF, OLIVA VL, BOUCHER LM, et al. Embolization of pulmonary arteriovenous malformations with amplatzer vascular plugs: safety and midterm effectiveness. J Vasc Interv Radiol 2010; 21: 649-56.

16.- SHOVLIN C L, GUTTMACHER A E, BUSCARINI E, FAUGHNAN M E, HYLAND R H, WESTERMANN $\mathrm{C} \mathrm{J}$, et al. Diagnostic criteria for hereditary hemorrhagic telangiectasia (Rendu-Osler-Weber syndrome). Am J Med Genet 2000; 91: 66-7.

17.- LITZLER PY, DOUVRIN F, BOUCHART F, TABLEY A, LEMERCIER E, BASTE J M, et al. Combined endovascular and video-assisted thoracoscopic procedure for treatment of a ruptured pulmonary arteriovenous fistula: Case report and review of the literature. J Thorac Cardiovasc Surg 2003; 126: 1204-7.

18.- AUSÍN HERRERO P, GÓMEZ-CARO ANDRÉS A, MORADIELLOS DÍEZ F J. [Spontaneous hemothorax due to rupture of a pulmonary artery aneurysm in Rendu-Osler-Weber syndrome]. Arch Bronconeumol 2004; 40: 603.

19.- KHAN A A, HUNT I, HAMDANE K, TAMBIAH J, DESHPANDE R P, REIDY J F. Massive pulmonary arteriovenous malformation presenting with tamponading haemothorax. Thorax 2007; 62: 836.

20.- SWIETLIK E, DOBOSZYNSKA A. Recurrence of arterio-venous malformations with life-threatening complications in a pregnant woman with hereditary teleangiectasia. J Physiol Pharmacol 2008; 59: 683-8.

21.- BERG A M, AMIRBEKIAN S, MOJIBIAN H, TROW T K, SMITH S J, WHITE RI Jr. Hemothorax due to rupture of pulmonary arteriovenous malformation: an interventional emergency. Chest 2010; 137: 705-7.

22.- FAUGHNAN M E, PALDA V A, GARCÍA-TSAO G, GEISTHOFF U W, MCDONALD J, PROCTOR D D, et al. International Guidelines for the Diagnosis and Management of Hereditary Hemorrhagic Telangiectasia. J Med Genet 2011; 48: 73-87.

23.- GOSSAGE J R, KANJ G. Pulmonary arteriovenous malformations. A state of the art review. Am J Respir Crit Care Med 1998; 158: 643-61.

24.- MORRELL N W. Screening for pulmonary arteriovenous malformations. Am J Respir Crit Care Med 2004; 169: 978-9.

25.- GONZÁLEZ S B, BUSQUETS J C, FIGUEIRAS R G, MARTÍN C V, POSE C S, DE ALEGRÍA A M, et al. Imaging arteriovenous fistulas. Am J Roentgenol 2009; 193: 1425-33.

26.- HU S Y, TSAI C H, TSAN Y T. Pulmonary arteriovenous malformation in Osler-Weber-Rendu syndrome. Eur J Cardiothorac Surg 2009; 36: 395.

27.- KRAEMER N, KROMBACH G A. Images in clinical medicine. Pulmonary arteriovenous fistula. N Engl J Med 2009; 360: 1769.

28.- TEMES R T, PARAMSOTHY P, ENDARA S A, WER- 
NLY J A. Resection of a solitary pulmonary arteriovenous malformation by video- assisted thoracic surgery. J Thorac Cardiovasc Surg 1998; 116: 878-9.

29.- SCHRÖDER C, FRÖHLICH G, HARMS C P, KLECKOW M, MACCHIARINI P. Fistulectomy as an alternative to segmentectomy for pulmonary arteriovenous fistula. J Thorac Cardiovasc Surg 2001; 122: 386-8.

30.- GEORGHIOU G P, BERMAN M, VIDNE B A, SAUTE M. Pulmonary arteriovenous malformation treated by lobectomy. Eur J Cardiothorac Surg 2003; 24: 328-30.

31.- LISCHKE R, SIMONEK J, STOLZ A, PAFKO P. Bilateral pulmonary arteriovenous malformations in patient with Rendu-Osler-Weber disease. Eur J Cardiothorac Surg 2004; 25: 461.

32.- FRAGA J C, FAVERO E, CONTELLI F, CANANI F. Surgical treatment of congenital pulmonary arteriovenous fistula in children. J Pediatr Surg 2008; 43: 1365-7.
33.- TAKAHAMA M, YAMAMOTO R, NAKAJIMA R, IZUMI N, TADA H. Surgery for multiple and diffuse pulmonary arteriovenous fistulas during childhood. Gen Thorac Cardiovasc Surg 2009; 57: 385-8.

34.- REYNAUD-GAUBERT M, THOMAS P, GAUBERT JY, PIETRI P, GARBE L, GIUDICELLI R, et al. Pulmonary arteriovenous malformations: lung transplantation as a therapeutic option. Eur Respir J 1999; 14: 1425-8.

35.- SVETLIZA G, DE LA CANAL A, BEVERAGGI E, GIACOIA A, RUIZ C, CARUSO E, et al. Lung transplantation in a patient with arteriovenous malformations. J Heart Lung Transplant 2002; 21: 506-8.

36.- GONZALEZ R, LAZO D, PRATS R, SANTOLAYA R, LINACRE V, RODRÍGUEZ P. Tratamiento quirúrgico de fistula arteriovenosa pulmonar en telangectasia hemorrágica hereditaria (Enfermedad de Rendu Osler Weber). Rev Chil Cir 2010; 62: 165-8.

Correspondencia a:

Dr. Roberto González Lagos

Instituto Nacional del Tórax, $4^{\circ}$ piso,

Sección de Cirugía de Tórax.

José Miguel Infante 717 ,

Providencia, Santiago, Chile.

Fono-fax: 056-02-5754997

E-mail: rgonzalezlagos@udec.cl 